\title{
Effects of Axillary Crutch Length on EMG Activity of the Trunk Muscles and Range of Motion of the Lumbar Spine, Pelvis, and Hip Joint in Healthy Men
}

\author{
Min-hyeok Kang ${ }^{1}$, MSc, PT, Jun-hyeok Jang ${ }^{2}$, PhD, PT, Tae-hoon Kim ${ }^{3}$, MPH, OT, \\ Jae-seop $\mathrm{Oh}^{4}, \mathrm{PhD}, \mathrm{PT}$ \\ ${ }^{1}$ Dept. of Rehabilitation Science, The Graduate School, Inje University, \\ ${ }^{2}$ Dept. of Physical Therapy, Haeundae Jaseng Hospital of Oriental Medicine, \\ ${ }^{3}$ Dept. of Occupational Therapy, Kaya University, \\ ${ }^{4}$ Dept. of Physical Therapy, College of Biomedical Science and Engineering, Inje University
}

\begin{abstract}
This study investigated the effects of axillary crutch length on trunk muscle activity and lumbo-pelvic-hip complex movements during crutch gait. Eleven healthy men participated in this study. The participants performed a three-point gait with optimal, shorter, and longer crutch lengths. Weight-bearing (WB) side was determined as the dominant leg side. The electromyography (EMG) activity of the bilateral rectus abdominis (RA) and erector spinae (ES) muscles and lumbo-pelvic-hip complex movements were monitored using a three-dimensional motion system with wireless surface EMG. Differences in the EMG activity of RA and ES muscles and range of motion (ROM) of lumbar spine, pelvis, and hip among conditions were analyzed using one-way repeated-measures analysis of variance, and a Bonferroni correction was conducted. There was less RA muscle activity on the WB side under the optimal crutch length condition compared with shorter and longer crutch length conditions $(p<.05)$. The EMG activity of the RA muscle on the non-WB side and ES muscle on the WB side were significantly decreased under the optimal crutch length condition compared with shorter crutch length condition $(\mathrm{p}<.05)$. No significant differences in the EMG activity of the ES muscle on the non-WB side and ROM of lumbo-pelvic-hip complex were found among conditions $(\mathrm{p}>.05)$. These findings indicate that the optimal crutch length improves the trunk muscle efficiency during crutch gait.
\end{abstract}

Key Words: Axillary crutch length; Electromyography; Range of motion; Three-point gait; Trunk muscles.

\section{Introduction}

Walking aids are commonly used to achieve an independent gait in injured people (Carpentier et al, 2010). Walking aids have the reported advantages of improving stability and balance, as well as minimizing weight-bearing (WB) on the affected leg (Faruqui and Jaeblon, 2010; O'sullivan and Schmitz, 2007). Among the various walking aids available (e.g, crutches, walkers, and canes), the axillary crutch is one of the most frequently recommended walking aids mainly for patients with musculoskeletal injury including fracture and occationally for patients with paraplegia (Bauer et al, 1991; Carpentier et al, 2010; Holder et al, 1993; Nyland et al, 2004).

The axillary crutch is comprised of an adjustable axillary bar and hand grip (Faruqui and Jaeblon, 2010). Several researchers have emphasized the importance of an optimal crutch length in preventing injury (Bauer et al, 1991; Raikin and Froimson, 1997; Veerendrakumar et al, 2001). Optimal crutch length is defined as having the crutch tips placed $15.2 \mathrm{~cm}$ an-

Corresponding author: Jae-seop Oh ysrehab@inje.ac.kr 
teriorly and laterally from the fifth toe, respectively, when the axillary bar is placed $5.1 \mathrm{~cm}$ below the axillary fold with approximately $25^{\circ}$ of elbow flexion in the standing position (Bauer et al, 1991). A shorter crutch length may induce poor posture (e.g, kyphosis) (Bauer et al, 1991; Kim et al, 1996), while a longer crutch length may be a risk factor for brachial plexus injury (Raikin and Froimson, 1997) and ulnar nerve palsy (Veerendrakumar et al, 2001).

To reduce the time required for measuring the $\mathrm{op}^{-}$ timal crutch length, various estimation techniques have been suggested, including: (1) $77 \%$ of the patient's height, (2) the patient's height minus $40.6 \mathrm{~cm}$, (3) the length between the olecranon of one arm and the middle finger of the other arm, (4) the length between the olecranon of one arm and the little finger of the other arm, (5) $77 \%$ of the arm span, (6) the arm span minus $40.6 \mathrm{~cm}$, and (7) the length between the axillary fold and the heel (Bauer et al, 1991; Kim et al, 1996). Bauer et al (1991) compared various estimation techniques and found that $71.9 \%$ of the patient's height plus $5.1 \mathrm{~cm}$ was the most accurate method in predicting the optimal crutch length.

It has been reported that inappropriate crutch length could induce kyphosis or trunk forward leaning posture (Bauer et al, 1991; Kim et al, 1996). Poor trunk postures have been stated as the risk factors for inefficient trunk muscle activity and lumbo-pelvic-hip complex movement during gait (Neumann, 2010; Perry and Burnfield, 2010). Yeung et al (2012) compared back muscle activity and lumbo-pelvic movement between gait with and without crutch in patients with spastic diplegia, and showed that EMG activity of back extensor muscles could be influenced by changes in kinematics of lumbo-pelvic segment in the sagittal plane during crutch gait. Yeung et al (2012) reported changes in trunk muscle activity and lumbar spine movement between gait with and without crutch, however, the authors did not demonstrate how crutch length affects back muscle activity and lumbar spine movement.
Although the importance of optimal crutch length has been reported in previous studies, there is a lack of consideration of the objective assessment, especially in trunk muscle activity and kinematics of lumbo-pelvic-hip complex when using optimal crutch length. Most previous studies have focused on the oxygen cost or perceived exertion during crutch gait (Holder et al, 1993; Mullis and Dent, 2000), and the importance of trunk muscle efficiency has been overlooked. It has been stated that shorter crutch length lead to kyphosis in the literature, but no study assessed objectively the influences of crutch length on the EMG activity of the trunk muscles and the kinematics of the lumbo-pelvic-hip complex during crutch gait.

The purpose of this study was to examine the effects of axillary crutch length on the EMG activity of the bilateral rectus abdominis (RA) and erector spinae (ES) muscles as well as the range of motion (ROM) of the lumbar spine, pelvis, and hip during crutch gait. Although no study reported how many patients suffer from inappropriate crutch length, considering frequently use of crutch in patient with musculoskeletal injury and neurologic disease, investigating the effects of the crutch length on trunk muscles activity and the kinematics of the lumbo-pelvic-hip complex during crutch gait will provide beneficial information to the clinician for designing crutch length for efficient crutch gait. We hypothesized that RA and ES muscle activity and the ROM of the lumbo-pelvic-hip complex would be decreased at an optimal crutch length compared with a shorter or longer than optimal crutch length during crutch gait.

\section{Methods}

\section{Subjects}

This experiment is performed from September 3 to 20, 2012, and eleven healthy men were recruited from Inje University, Korea for this study. 


\begin{tabular}{cc}
\hline \hline Variables & Mean \pm SD \\
\hline Age (yrs) & $23.2 \pm 2.4$ \\
Weight $(\mathrm{kg})$ & $63.9 \pm 5.3$ \\
Height $(\mathrm{cm})$ & $173.5 \pm .9$ \\
\hline
\end{tabular}

Individuals were excluded from this study if they had a history of cardiopulmonary disease or surgery in the lower extremities or lower back. Table 1 shows the general information of the participants. Prior to participation, all subjects provided written, informed consent.

\section{Instruments and Measurements}

\section{Surface Electromyography}

EMG data from the bilateral RA and ES muscles were monitored using a Delsys Trigno Wireless EMG System (Delsys Trigno Wireless EMG System, Delsys Inc., Boston, USA). The EMG signals were bandwidth-filtered at $20 \sim 450 \mathrm{~Hz}$, and the EMG data were converted to the root mean square (RMS). To minimize skin resistance, the body hair was shaved and skin was swabbed with $70 \%$ isopropyl alcohol-soaked cotton prior to electrode attachment. Electrodes were placed bilaterally on the RA muscles, $3 \mathrm{~cm}$ from the umbilicus in the direction of the muscle fibers, while electrodes were attached to the ES muscles bilaterally, $2 \mathrm{~cm}$ apart from the third lumbar spinous process in the direction of the muscle fibers (Criswell, 2010). The maximum voluntary isometric contraction (MVIC) values for the RA and ES muscles were measured for normalization. Two $5 \mathrm{~s}$ MVIC tests for each muscle were performed by a manual muscle test as described by Kendall et al (2005). To test the ES muscles, the examiner stabilized the legs while the participants raised his trunk upward in the prone position, against gravity, with hands behind the head. For measurement of MVIC of RA muscles, participants positioned supine on the table with hands behind the head and knees straight. Participants performed curl-up until scapulae clear table, and then maximum manual resistance was ap- plied to shoulders. The average muscle activity for the middle $3 \mathrm{~s}$ of the two trials was used for the normalization of each muscle.

\section{Kinematics}

Kinematic data from the lumbar spine, pelvis, and hip were recorded using a VICON Motion Analysis System (VICON Motion Analysis System, Vicon Motion Systems Ltd., Oxford, UK) with eight MX-T10 cameras at a sampling rate of $100 \mathrm{~Hz}$. To measure pelvis and hip movement, 16 reflective markers were placed on subjects' bilateral anterior and posterior superior iliac spines, lateral thighs, lateral knees, tibias, lateral malleoli, second metatarsal heads, and posterior calcaneuses based on the VICON Plug-in-Gait marker set. To measure lumbar spine movement, an additional four markers were attached to the first and second lumbar spinous processes and $2 \mathrm{~cm}$ apart from second lumbar spinous process, on both sides. As flexion and extension of lumbar spine comes from combined movement of both lumbar spine and pelvis referred to lumbo-pelvic rhythm (Neumann, 2010), we measured not only lumbar motion but also pelvic motion in the present study. Angular value of the lumbar spine, pelvis, and hip were calculated using the Cardan angle (Kadaba et al, 1990).

\section{Axillary Crutch Length}

For this study, two length-adjustable axillary crutches (Axillary Crutch, Samboo Medical Co., Ltd., Daejeon, Korea) were used. The unit of adjustable length of axillary crutch is $3 \mathrm{~cm}$. The examiner determined the optimal crutch length, shorter crutch length, and longer crutch length for each participant. The optimal crutch length was calculated as $71.9 \%$ of each participant's height plus $5.1 \mathrm{~cm}$, as suggested 


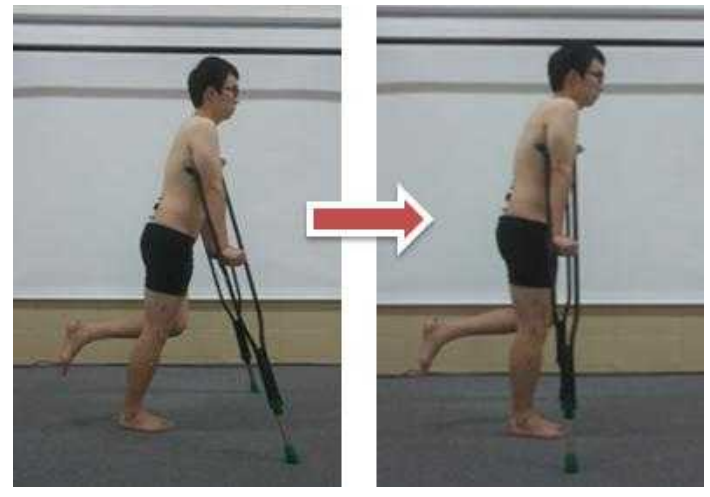

Figure 1. Step-to three-point gait.

by Bauer et al (1991). In our pilot study, participants had difficulty in heel strike when they used $6 \mathrm{~cm}$ longer crutch length than optimal crutch length. In the normal gait pattern, initial foot contact is generated by heel strike (Perry and Burnfield, 2010). Therefore, the shorter and longer crutch lengths were defined as the optimal crutch length minus 3 $\mathrm{cm}$ and plus $3 \mathrm{~cm}$, respectively, in this study. Mean optimal crutch length for participants was $129.55 \pm 1.21$ $\mathrm{cm}$ in the present study.

\section{Procedures}

Prior to the experiment, dominant leg side of participant was determined by asking preferred leg side when kicking a ball (Fagenbaum and Darling, 2003). For the step-to three-point gait, the participant's body weight was borne on the dominant leg and the opposite leg was non-WB while both crutches were advanced. The body weight was shifted onto the crutches through the upper extremities, and the dominant leg advanced to a line between the crutches (Figure 1). This cycle was repeated for the step-to three-point gait. All participants practiced the step-to three-point gait for approximately $15 \mathrm{~min}$ for each crutch length condition. The familiarization period was complete when participants performed initial foot contact by heel strike without discomfort during crutch gait. The order of the crutch length was randomized, and the step-to three-point gait was repeated three times at a self-determined speed under each condition with rest periods of 1 min between trials and $3 \mathrm{~min}$ between crutch length conditions. The step-to three-point gait cycle was performed for five cycles for one trial, and the third cycle of each trial was used for data analysis.

\section{Data Analysis}

Nexus (Nexus ver. 1.5, Vicon Motion Systems Ltd., Oxford, UK) software was used to calculate the $\mathrm{ROM}$ of the lumbar spine, pelvis, and hip on the WB side. In the present study, ROM was defined as the change in the maximum-to-minimum angular value of each segment in the sagittal plane. The mean value of the ROM of the lumbar spine, pelvis, and hip of three trials was determined for kinematic data analysis.

The mean RMS data for RA and ES muscle activity during the crutch gait cycle were expressed as the \%MVIC. The mean \%MVIC value of three trials was determined for EMG data analysis.

\section{Statistical Analysis}

A one-way repeated-measures analysis of variance was used to determine the main effects of crutch length on the kinematic and EMG data. The post hoc comparative analysis of the three different crutch lengths was performed with Bonferroni correction. The level of statistical significance was set at $a=.05$. The data were analyzed using SPSS ver. 17.0 software.

\section{Results}

EMG activity of the bilateral RA muscles and ES muscle on the WB side was significantly different among the three crutch lengths $(\mathrm{p}<.05)$ (Table 2). Post hoc analysis revealed that the EMG activity of the RA muscle on the WB side was decreased significantly in optimal crutch length condition compared with shorter and longer crutch length conditions, while RA muscle activity on the non-WB side and ES muscle activity on the WB side was decreased 


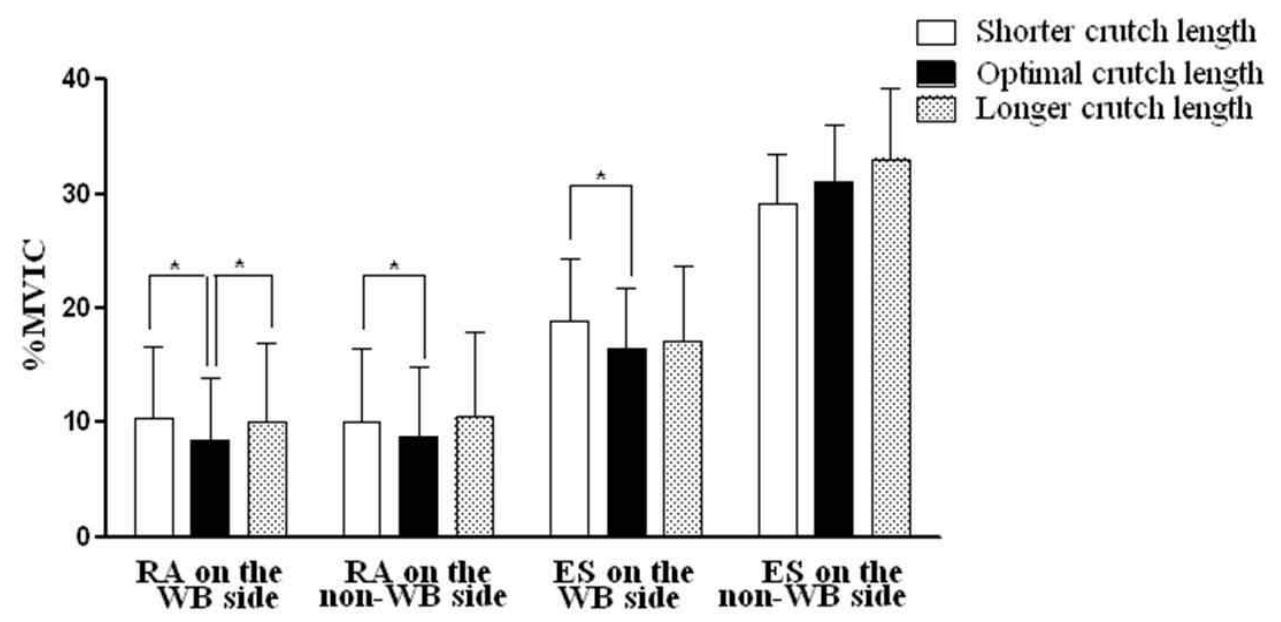

Figure 2. Comparison of trunk muscle activity among three different crutch lengths (RA: rectus abdominis, ES: erector spinae, WB: weight-bearing, \%MVIC: \%maximum voluntary isometric contraction, $\left.{ }^{*} \mathrm{p}<.05\right)$.

significantly in optimal crutch condition compared with only shorter crutch length $(\mathrm{p}<.05)$ (Figure 2$)$.

No significant differences in ES activity on the non-WB side or ROM of the lumbar spine, pelvis, and hip were observed among the three crutch length conditions ( $>$.05) (Table 3) (Figure 3).

\section{Discussion}

Improper axillary crutch length has been reported to lead to poor trunk posture (Kim et al, 1996; Raikin and Froimson, 1997; Veerendrakumar et al, 2001). However, most studies have focused on the methods in predicting optimal crutch length, no study performed objective assessment whether the crutch length influences trunk muscles activity and ROM of lumbo-pelvic-hip complex during crutch gait. Thus, we examined the influences of crutch length on trunk muscle activity and the ROM of the lumbar spine, pelvis, and hip during crutch gait.

Our results showed significant differences in EMG activity of the bilateral RA muscles among the three conditions $(\mathrm{p}<.05)$. In general, the trunk muscles are involved in trunk stability during gait (Neumann, 2010; Perry and Burnfield, 2010; Yeung et al, 2012). It has been reported that the RA muscles help stabilize the lower back and pelvis to provide stable fix ${ }^{-}$ ation for hip flexion during gait (Neumann, 2010). The previous study by Olson (2010) showed increase in RA muscle activity during swing phase of gait, which supported the role of RA muscles in trunk stability during gait. Olson (2010) suggested that RA muscle activity was increased to sustain trunk stability during swing phase of gait, although the changes in EMG activity of RA was low. In our results, there was significant decrease in RA muscle activity on the WB side under the optimal crutch

Table 2. EMG activity of RA and ES muscles during crutch gait at the three crutch lengths (Unit: \%MVIC)

\begin{tabular}{ccccc}
\hline \hline Muscles & Shorter crutch length & Optimal crutch length & Longer crutch length & $\mathrm{p}$ \\
\hline $\mathrm{RA}^{\mathrm{a}}$ on the $\mathrm{WB}^{\mathrm{b}}$ side & $10.30 \pm 6.40^{\mathrm{d}}$ & $8.48 \pm 5.49$ & $10.09 \pm 6.79$ & .011 \\
$\mathrm{RA}$ on the non $-\mathrm{WB}$ side & $10.09 \pm 6.41$ & $8.77 \pm 6.05$ & $10.50 \pm 7.44$ & .046 \\
$\mathrm{ES}^{\mathrm{c}}$ on the WB side & $18.89 \pm 5.49$ & $16.50 \pm 5.34$ & $17.05 \pm 6.71$ & .015 \\
$\mathrm{ES}$ on the non-WB side & $29.14 \pm 4.29$ & $31.03 \pm 5.03$ & $33.05 \pm 6.23$ & .135 \\
\hline
\end{tabular}

${ }^{a}$ rectus abdominis, ${ }^{b}$ weight-bearing, ${ }^{c}$ erector spinae, ${ }^{\mathrm{d}}$ mean \pm standard deviation. 


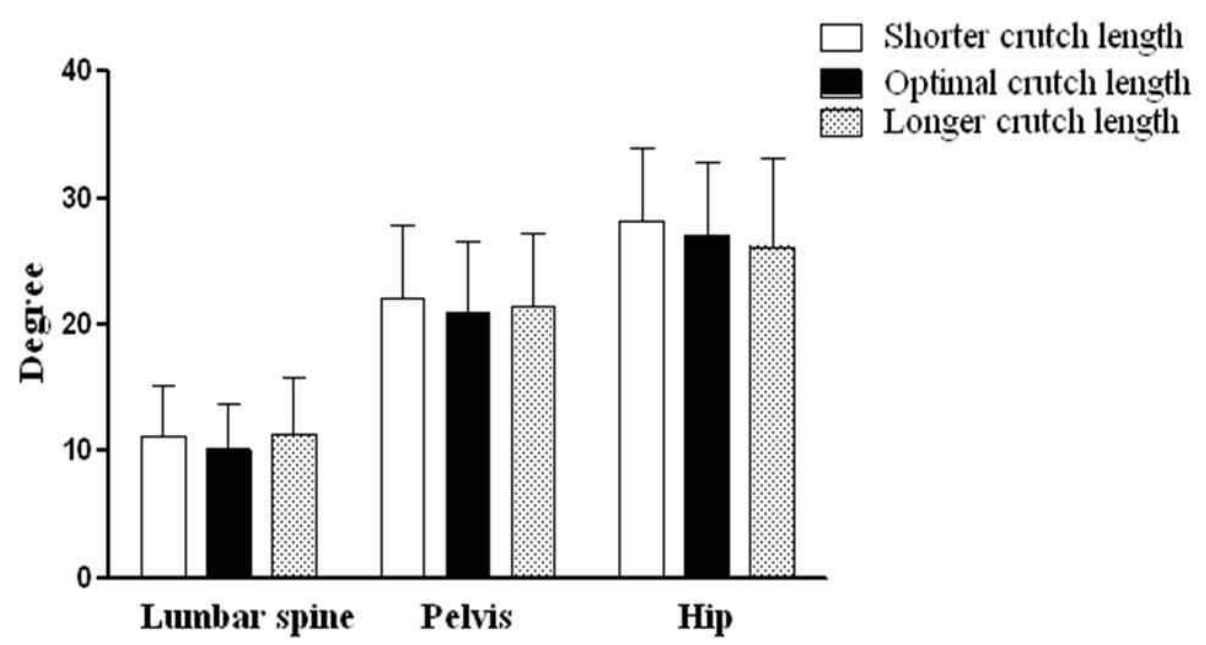

Figure 3. Comparison of range of motion among three different crutch lengths.

length condition compared with shorter and longer crutch length conditions $(\mathrm{p}<.05)$. It is inferred that optimal crutch length provides proper trunk stability, which may substitute for the role of RA muscles during crutch gait. Even though significant decrease in EMG activity of RA muscle on the non-WB side was found under the optimal crutch length condition compared with only shorter crutch length condition $(p<.05)$, there was a tendency for decreased RA muscle activity on the non-WB side under the optimal crutch length condition compared with longer crutch length condition $(\mathrm{p}=.072)$. It is considered that no significant difference between two conditions is caused by small sample size in the present study.

Compare with shorter crutch length condition, under the optimal crutch length condition, EMG activity of ES muscle on the WB side was decreased significantly in the present study $(\mathrm{p}<.05)$. ES muscles activity is important factor in energy con- servation during gait, as ES muscles control the forward angular displacement of the trunk (Perry and Burnfield, 2010; Yeung et al, 2012). Yeung et al (2012) suggested that extension moment generated by crutches decreased ES muscle activity, which might reduce energy cost during crutch gait. Based on previous findings, the trunk stability provided by crutches may be greater under the optimal crutch length condition compared with the shorter crutch length condition, which may decrease the EMG activity of the ES muscle on the WB side in our study. It is possible that the decreases in EMG activity of the trunk muscles in the present study indicate improvements in gait performance and efficiency of the trunk muscles during crutch gait.

In contrast to the bilateral RA muscles and ES muscle on the WB side, there was no significant difference in ES activity on the non-WB side among the conditions ( $\mathrm{p}>$.05). During the gait cycle, the ES

Table 3. Range of motion of lumbar spine, pelvis, and hip during crutch gait at the three crutch lengths

(Unit: ${ }^{\circ}$ )

\begin{tabular}{ccccc}
\hline \hline Segments & Shorter crutch length & Optimal crutch length & Longer crutch length & $\mathrm{p}$ \\
\hline Lumbar spine & $11.23 \pm 4.03^{\text {a }}$ & $10.11 \pm 3.70$ & $11.36 \pm 4.42$ & .247 \\
Pelvis & $22.10 \pm 5.79$ & $20.98 \pm 5.59$ & $21.39 \pm 5.79$ & .715 \\
Hip & $28.21 \pm 5.72$ & $27.09 \pm 5.82$ & $26.15 \pm 7.00$ & .255 \\
\hline
\end{tabular}

\footnotetext{
${ }^{a}$ mean \pm standard deviation.
} 
muscle has two definite periods of activity (Levangie and Norkin, 2005; Neumann, 2010). The EMG activity of the ES muscle is changed noticeably during the loading response and pre-swing phases of the gait cycle (Neumann, 2010; Perry and Burnfield, 2010). As the participants in the present study performed the step-to three-point gait with bearing weight on the dominant leg, a relatively steady muscle activity was generated in the ES muscle on the non-WB side compared with the WB side. Although mean RMS data used for data analysis in this study, we consider that instantaneously changed muscle activity in the loading response and pre-swing phases may influence mean ES muscle activity during crutch gait cycle. Therefore, crutch length may affect significantly ES muscle activity on the WB side when the demands on the ES muscle on the WB side are instantaneously changed, which may result in no significant change in the ES muscle on the non-WB side during crutch gait.

It has been reported that RA muscle activity was steady at approximately 2 \%MVIC (Olson, 2010), while mean EMG activity of ES muscles was 4.26 \%MVIC during gait (Barton et al, 2009). Compare with previous findings, EMG activity of RA and ES muscles are higher during crutch gait in our study. It may imply that more trunk muscles activity are required during crutch gait compared with gait without crutch. Therefore, the use of optimal crutch length needs to be suggested to improve efficiency of trunk muscles during crutch gait.

Even though we hypothesized that the kinematics of the lumbo-pelvic-hip complex would be affected by the crutch length during crutch gait, the ROM in the lumbar spine, pelvis, and hip did not differ among the three crutch length conditions ( $p>05$ ). It has been stated that crutches that are too short can cause an inappropriate body posture such as $\mathrm{ky}^{-}$ phosis (Bauer et al, 1991; Kim et al, 1996). In this study, the shorter crutch length and longer crutch length were defined as $3 \mathrm{~cm}$ shorter and longer than the optimal crutch length, respectively. It is inferred that the differences in crutch length among the three conditions were not sufficient to cause significant differences in the ROM of the lumbar spine, pelvis, and hip during crutch gait. In addition, the unit of adjustable length of crutch was $3 \mathrm{~cm}$ in the present study. Considering that $6 \mathrm{~cm}$ longer than optimal crutch length induced abnormal crutch gait pattern in our pilot study, smaller unit of adjustable length of crutch is needed to determine how crutch length influence ROM of lumbo-pelvic-hip complex during crutch gait. Another possible explanation is that all participants consist of healthy men in this study. It is considered that healthy subjects may have ability to maintain proper trunk posture in spite of difference in crutch length.

This study has a number of limitations. First, only healthy men were recruited as subjects. Previous study suggested that the fears of incomplete recovery in patients may influence mastery of use of crutch, which is related to fear of falling (Griffiths and Jordan, 1998). In addition, differences in kinematics of lower extremity and gait parameter such as gait velocity and stride length were observed between women and men during gait (Levangie and Norkin, 2005). Based on previous studies, it is possible that fear of falling and gender difference would influence biomechanics of trunk and lower $\mathrm{ex}^{-}$ tremities during crutch gait. Therefore, future studies must include women as well as patients who need to crutch for walking. Second, we used the step-to three-point gait for this study. Investigating other crutch gait patterns may help explain the effects of crutch length on the biomechanics of the trunk or lower extremities. Third, there is some difficulty in generalizing our results because of the small sample size. Fourth, it has difficulty in defining independent pelvic movement. As pelvic segment is linked to lumbar spine and hip, movement of adjacent segment could influence pelvic movement. Lastly, we did not measure peak EMG data during crutch gait. In the future study, analysis of peak EMG data will provide useful information about the roles of trunk muscles 
in the phase of crutch gait.

\section{Conclusion}

We examined the influences of crutch length on EMG activity of RA and ES muscles and ROM of lumbo-pelvic-hip complex during step-to three-point gait in healthy men. Participants performed step-to three-point gait using three different crutch lengths in the randomized order, and trunk muscle activity and ROM of lumbar spine, pelvis, and hip during crutch gait were analyzed. We found the EMG activity of the RA muscle on the WB side was significantly less under optimal crutch length condition than under the other crutch length conditions $(\mathrm{p}<.05)$. RA muscle activity on the non-WB side and ES muscle activity on the WB side were significantly decreased under the optimal crutch length condition compared with shorter crutch length condition $(p<.05)$. However, crutch length did not significantly change the EMG activity of the ES muscle on the non-WB side or the ROM of the lumbar spine, pelvis, and hip during crutch gait ( $p>05)$. These results suggest that optimal crutch length should be considered to improve the efficiency of the trunk muscles during crutch gait.

\section{References}

Barton CJ, Coyle JA, Tinley P. The effect of heel lifts on trunk muscle activation during gait: A study of young healthy females. J Electromyogr Kinesiol. 2009;19(4):598-606.

Bauer DM, Finch DC, McGough KP, et al. A comparative analysis of several crutch-length-estimation techniques. Phys Ther. 1991;71(4):294-300.

Carpentier C, Font-Llagunes JM, Kövecses J. Dynamics and energetics of impacts in crutch walking. J Appl Biomech. 2010;26(4):473-483.

Criswell E. Introduction to Surface Electromyography. 2nd ed. Sudbury, MA, Jones and Bartlett Publishers, 2010:342-348.

Fagenbaum R, Darling WG. Jump landing strategies in male and female college athletes and the implications of such strategies for anterior cruciate ligament injury. Am J Sports Med. 2003;31(2): 233-240.

Faruqui SR, Jaeblon T. Ambulatory assistive devices in orthopaedics: Uses and modifications. J Am Acad Orthop Surg. 2010;18(1):41-50.

Griffiths H, Jordan S. Thinking of the future and walking back to normal: An exploratory study of patients' experiences during recovery from lower limb fracture. J Adv Nurs. 1998;28(6): 1276-1288.

Holder CG, Haskvitz EM, Weltman A. The effects of assistive devices on the oxygen cost, car- $^{-}$ diovascular stress, and perception of nonweight-bearing ambulation. J Orthop Sports Phys Ther. 1993;18(4):537-542.

Kadaba MP, Ramakrishnan HK, Wootten ME. Measurement of lower extremity kinematics during level walking. J Orthop Res. 1990;8(3): 383-392.

Kendall FP, McCreary EK, Provance PG, et al. Muscles: Testing and Function with Posture and Pain. 5th ed. Baltimore, MD, Williams \& Wilkins, 2005:176-203.

Kim MJ, Park YS, Yi $\mathrm{CH}$, et al. A comparative analysis between several crutch-length-estimation techniques and ideal crutch length. Phys Ther Kor. 1996;3(1):24-31.

Levangie PK, Norkin CC. Joint Structure and Function: A Comprehensive Analysis. 4th ed. Philadelphia, PA, F.A. Davis Company, 2005: 517-547.

Mullis R, Dent RM. Crutch length: Effect on energy cost and activity intensity in non-weight-bearing ambulation. Arch Phys Med Rehabil. 2000;81(5):569-572.

Neumann DA. Kinesiology of the Musculoskeletal System: Foundations for rehabilitation. 2nd ed. 
St. Louis, MO, Mosby, 2010:627-650.

Nyland J, Bernasek T, Markee B, et al. Comparison of the easy strutter functional orthosis system and axillary crutches during modified 3-point gait. J Rehabil Res Dev. 2004;41(2):195-206.

Olson MW. Trunk extensor fatigue influences trunk muscle activities during walking gait. J Electromyogr Kinesiol. 2010;20(1):17-24.

O'sullivan SB, Schmitz TJ. Physical Rehabilitation. 5th ed. Philadelphia, PA, F.A. Davis Co., 2007:545-549.

Perry J, Burnfield JM. Gait Analysis: Normal and pathological function. 2nd ed. Thorofare, NJ, SLACK Inc., 2010:121-127.

Raikin S, Froimson MI. Bilateral brachial plexus compressive neuropathy (crutch palsy). J Orthop Trauma. 1997;11(2):136-138.

Veerendrakumar M, Taly AB, Nagaraja D. Ulnar nerve palsy due to axillary crutch. Neurol India. 2001;49(1):67-70.

Yeung EH, Chow DH, Su IY. Kinematic and electromyographic studies on unaided, unilateral and bilateral crutch walking in adolescents with spastic diplegia. Prosthet Orthot Int 2012;36(1):63-70.

This article was received October 26, 2012, was reviewed October 26, 2012, and was accepted December 17, 2012 